\title{
A convenient procedure for the synthesis of allyl and benzyl ethers from alcohols and phenols
}

\author{
H SURYA PRAKASH RAO* and S P SENTHILKUMAR \\ Department of Chemistry, Pondicherry University, Pondicherry 605014 , \\ India \\ e-mail: hspr@satyam.net.in
}

MS received 29 December 2000

\begin{abstract}
Allyl and benzyl ethers of alcohols can be prepared conveniently and in high yield with allyl and benzyl bromide in the presence of solid potassium hydroxide without use of any solvent. Phenols can be converted to allyl ethers but are inert to benzylation under above conditions.
\end{abstract}

Keywords. Allyl and benzyl ethers; potassium hydroxide; solvent-free conditions.

\section{Introduction}

Allyl and benzyl groups are commonly employed for the protection of alcohol and phenol moieties for ease of synthesis and convenient deprotection. Allyl and benzyl ethers are also intermediates in sigmatropic rearrangement reactions such as the Claisen and the Cope rearrangements. Allyl ethers can be prepared from the corresponding alcohols using several reagents such as allyl bromide ${ }^{1}$, allyl carbonate ${ }^{2,3}$, allyl ethyl carbonate ${ }^{4}$ etc. Similarly, benzyl ethers can be prepared using benzyl bromide ${ }^{5}$, benzyl iodide ${ }^{6}$, phenyl diazomethane ${ }^{7}$ etc. Among the above, allyl bromide and benzyl bromide in combination with a base in a suitable solvent medium are frequently employed for the generation of allyl and benzyl ethers respectively. In continuation of our studies on the Williamson synthesis (preparation of ethers) in solvent-free environments ${ }^{8}$, we have considered the protection of alcohol with allyl and benzyl groups under similar conditions. We found that alcohol protection can be carried out conveniently and efficiently with allyl and benzyl bromides, with solid potassium hydroxide pellets without the use of any solvent (scheme 1). Even though benzyl protection on a carbohydrate substrate was previously carried out with benzyl chloride in the presence of potassium hydroxide pellets ${ }^{9}$, the scope of the reaction was not fully explored. While our work was in progress, Bogdal and coworkers recently reported ${ }^{10}$ solvent-free allyl and benzyl ether preparation using a combination of potassium carbonate and potassium hydroxide bases in the presence of tetrabutylammonium bromide under microwave irradiation. However, the reaction requires drastic conditions such as high temperature and specialized apparatus. Moreover, their study was limited to the preparation of ethers from alcohols.

*For correspondence 


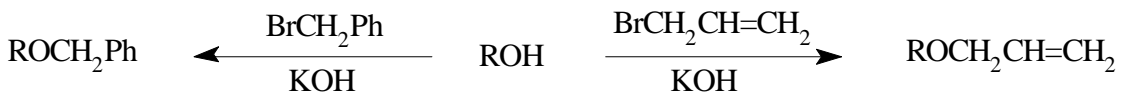

\section{Scheme 1.}

To prepare allyl ethers, the alcohol/phenol and the allyl bromide were stirred with solid potassium hydroxide pellets in the presence of 5 mole percent of tetrabutylammonium iodide (TBAI), a phase transfer catalyst (PTC), for the specified time at room temperature (table 1) for completion of the reaction. Excess allyl bromide was removed through distillation followed by purification through chromatography on a short column of silica gel. Similarly, benzyl ethers were prepared from alcohols and benzyl bromide (table 1). It is evident from a perusal of the table that, in general, allyl ether formation is faster and better yields can be obtained in comparison with benzyl ethers. It is seen that a wide range of substrates displaying different structural features one readily converted to allyl ethers. We also found that the reaction with allyl bromide can be performed conveniently on a molar scale and excess reagent can be recovered through simple distillation. We explored the efficacy of the allyl protection of cholesterol in the presence of different PTCs such as TBAI, benzyltriethylammonium bromide and 18-crown-6. We found that in the presence of PTC, particularly TBAI, time taken for completion of the allyl protection is about three time less than the time taken for the reaction without PTC. However, for benzyl ether preparation no such beneficial effect of the PTC is observed. Interestingly, benzylation of menthol does not take place under the present conditions. In a competitive experiment, when cholesterol and menthol mixture are subjected to benzylation cholesterol is completely converted to its benzyl ether in $16 \mathrm{~h}$ whereas

Table 1. Conversion of alcohols and phenols to allyl and benzyl ethers.

\begin{tabular}{|c|c|c|c|}
\hline \multicolumn{4}{|c|}{$\mathrm{ROCH}_{2} \mathrm{CH}=\mathrm{CH}_{2} \leftarrow \mathrm{ROH} \rightarrow \mathrm{ROCH}_{2} \mathrm{C}_{6} \mathrm{H}_{5}$} \\
\hline No. & $\mathrm{ROH}$ & $\begin{array}{c}\text { Allyl ether; } \\
\text { time (h) (yield \%) }\end{array}$ & $\begin{array}{c}\text { Benzyl ether; } \\
\text { time (h) (yield \%) }\end{array}$ \\
\hline 1 & $\mathrm{CH}_{3}\left(\mathrm{CH}_{2}\right)_{8} \mathrm{CH}_{2} \mathrm{OH}$ & $2 \cdot 5(95)$ & $18(94)$ \\
\hline 2 & $\mathrm{C}_{6} \mathrm{H}_{5} \mathrm{CH}_{2} \mathrm{OH}$ & $4 \cdot 5(96)$ & $35(81)$ \\
\hline 3 & Geraniol & $5(96)$ & $18(91)$ \\
\hline 4 & Nerol & $5 \cdot 5(93)$ & $20(93)$ \\
\hline 5 & Menthol & $5 \cdot 5(90)$ & - \\
\hline 6 & Cholesterol & $7(98)$ & $16(86)$ \\
\hline 7 & & $3(95)$ & $20(94)$ \\
\hline 8 & & $3 \cdot 5(94)$ & $36(92)$ \\
\hline 9 & $\mathrm{C}_{6} \mathrm{H}_{5} \mathrm{OH}$ & $14(89)$ & - \\
\hline 10 & $p-\mathrm{CH}_{3} \mathrm{C}_{6} \mathrm{H}_{4} \mathrm{OH}$ & $15(85)$ & - \\
\hline
\end{tabular}


menthol is recovered unreacted, indicating selectivity due to subtle changes in steric environment. Phenolic hydroxy group is also found to be completely inert for benzylation under present conditions (entry 8, 9, table 1 ).

Thus, we have delineated a convenient procedure for the synthesis of allyl and benzyl ethers. In view of the enormous importance of allyl and benzyl protecting groups in organic synthesis we hope that the procedure described in this paper will find routine use.

\section{Experimental}

\subsection{General conditions}

Allyl bromide (Fluka) and benzyl bromide (E Merck) were freshly distilled before use. Purification of the products was carried out on a short silica gel column (100-200 mesh, Acme chemicals) using increasing percentage of ethyl acetate in hexane as elutant. The IR spectra were recorded on a Jasco FT-IR spectrometer. The ${ }^{1} \mathrm{H}$ NMR spectra were recorded on a Jeol $400 \mathrm{MHz}$ instrument as $\mathrm{CDCl}_{3}$ solutions with TMS as internal standard unless otherwise specified. The chemical shift values are given in (ppm) units relative to TMS. The product ethers were characterized by comparing spectral data of known compounds described in the literature or procured from commercial sources.

\subsection{Representative procedure for the synthesis of allyl ethers}

2.2a Synthesis of allyl decyl ether: Conversion of 1-decanol to its allyl ether is described as a representative example (entry 1, table 1). A mixture of 1-decanol (166 mg, $1.05 \mathrm{mmol})$, allyl bromide $(0.35 \mathrm{ml}, 4.04 \mathrm{mmol}), \mathrm{KOH}$ (1 pellet, $\approx 120 \mathrm{mg}, 2 \mathrm{mmol})$ and TBAI $(5 \mathrm{~mol} \%)$ was stirred at room temperature for $16 \mathrm{~h}$ (completion of the reaction was monitored by TLC). After completion of the reaction $(2.5 \mathrm{~h})$, the mixture excluding the $\mathrm{KOH}$ pellet was distilled under reduced pressure to remove excess allyl bromide and then loaded on a pad of silica gel (100-200 mesh, $1 \times 5 \mathrm{~cm}$ column) and eluted with hexane:ethyl acetate (95:5) to yield allyl decyl ether ${ }^{10}$ in $95 \%$ yield. IR (neat) 1100 , $1660 \mathrm{~cm}^{-1} ;{ }^{1} \mathrm{H}$ NMR: $\delta 5 \cdot 87-5.95(m, 1 \mathrm{H}), \delta 5 \cdot 15-5 \cdot 24(m, 2 \mathrm{H}), \delta 3.97(d, J=5.37 \mathrm{~Hz}$, $2 \mathrm{H}), \delta 3.41(t, J=13.67 \mathrm{~Hz}, 2 \mathrm{H}), \delta 1.6(m, 2 \mathrm{H}), \delta 1.26(\operatorname{broad} s, 14 \mathrm{H}), \delta 0.89(t$, $J=13 \cdot 2 \mathrm{~Hz}, 3 \mathrm{H})$.

2.2b Allyl benzyl ether (entry 2): Benzyl alcohol (108 mg, $1 \mathrm{mmol}$ ) was converted to allyl benzyl ether ${ }^{11}$ in $96 \%$ yield in $4.5 \mathrm{~h}$. IR (neat): $1630,1090 \mathrm{~cm}^{-1} ;{ }^{1} \mathrm{H}$ NMR: $\delta 7 \cdot 34$ $(m, 5 \mathrm{H}), \delta 5 \cdot 9-6 \cdot 0(m, 1 \mathrm{H}), \delta 5 \cdot 17-5 \cdot 35(m, 2 \mathrm{H}), \delta 4.5(s, 2 \mathrm{H}), \delta 4.04(d, J=1.46 \mathrm{~Hz}$, $2 \mathrm{H})$.

2.2c Allyl geranyl ether (entry 3): Geraniol (154 mg, $1 \mathrm{mmol}$ ) was converted to its allyl ether in $96 \%$ yield in $5 \mathrm{~h}$. IR (neat) $1660 \mathrm{~cm}^{-1} ;{ }^{1} \mathrm{H} \mathrm{NMR}\left(60 \mathrm{MHz} ; \mathrm{CDCl}_{3}: \mathrm{CCl}_{4} ; 1: 1\right)$ : $\delta 5 \cdot 6-5 \cdot 7(m, 1 \mathrm{H}), \delta 5 \cdot 4(m, 1 \mathrm{H}), \delta 5 \cdot 1-5 \cdot 2(m, 3 \mathrm{H}), \delta 3.97(b r s, 4 \mathrm{H}), \delta 2 \cdot 1(b r s, 4 \mathrm{H})$, $\delta 1.72(s, 3 \mathrm{H}), 1 \cdot 64(\mathrm{~s}, 6 \mathrm{H})$.

2.2d Allyl neryl ether (entry 4): Nerol (154 mg, $1 \mathrm{mmol})$ was converted to its allyl ether ${ }^{12}$ in $93 \%$ yield in $5.5 \mathrm{~h}$. IR (neat) $1660 \mathrm{~cm}^{-1} ;{ }^{1} \mathrm{H}$ NMR: $\delta 5 \cdot 85-5.94(m, 1 \mathrm{H}), \delta 5 \cdot 36$ $(t, J=13.7 \mathrm{~Hz}, 2 \mathrm{H}), \delta 5.09-5.28(m, 2 \mathrm{H}), \delta 3.93-3.96(m, 4 \mathrm{H}), \delta 2.07(s, 4 \mathrm{H}), \delta 1.74$, $1 \cdot 67,1 \cdot 6$ (three $s, 3 \times 3 \mathrm{H})$. 
2.2e Allyl menthyl ether (entry 5): Menthol (156 mg, $1 \mathrm{mmol}$ ) was converted to allyl menthyl ether ${ }^{13}$ in $90 \%$ yield in $5.5 \mathrm{~h} .[\alpha]_{\mathrm{D}}{ }^{27}=-53 \cdot 13^{\circ}$, IR (neat): $1630,1170 \mathrm{~cm}^{-1}$, ${ }^{1} \mathrm{H}$ NMR: $\delta 5 \cdot 87-5 \cdot 96(m, 1 \mathrm{H}), \delta 5 \cdot 11-5 \cdot 3(m, 2 \mathrm{H}), \delta 3 \cdot 85-4 \cdot 15(m, 3 \mathrm{H}), \delta 3.05(m, 1 \mathrm{H})$, $\delta 1.22-2.25(m, 6 \mathrm{H}), \delta 0.91(d, J=6.84 \mathrm{~Hz}, 3 \mathrm{H}) \delta 0.89(d, J=7.33 \mathrm{~Hz}, 3 \mathrm{H}), \delta 0.77(d$, $J=6 \cdot 84 \mathrm{~Hz}, 3 \mathrm{H})$.

2.2f Allyl cholesteryl ether (entry 6): Cholesterol (194 mg, $0.5 \mathrm{mmol}$ ) was converted to allyl cholesteryl ether ${ }^{14}$ in $96 \%$ yield in 7 h. M.p. $68^{\circ} \mathrm{C}[\alpha]_{D}=-21 \cdot 6^{\circ}$, IR (neat): 1647 , $1138 \mathrm{~cm}^{-1}$; ${ }^{1} \mathrm{H}$ NMR: $\delta 5 \cdot 76-6.06(\mathrm{~m}, 1 \mathrm{H}), \delta 5 \cdot 35(\mathrm{br} d, J=6.0 \mathrm{~Hz}, 1 \mathrm{H}), \delta 5 \cdot 06-5 \cdot 23(\mathrm{~m}$, $2 \mathrm{H}), \delta 4.03(d, J=5.78 \mathrm{~Hz}, 2 \mathrm{H}), \delta 2 \cdot 3-3.43(m, 1 \mathrm{H}) \delta 0.86-2.28(m, 28 \mathrm{H}), \delta 0.98(s, 3 \mathrm{H})$, $\delta 0.75-0.95(b r d, 9 \mathrm{H}), 0.63(s, 3 \mathrm{H})$.

2.2g 3-O-allyl-1,2:4,5-di-O-isopropylidenefructopyranose (entry 7): The fructose diacetonide (260 mg, $1 \mathrm{mmol}$ ) was converted to the corresponding allyl ether in $95 \%$ yield as a colourless oil in $3 \mathrm{~h}$. $[\alpha]_{\mathrm{D}}=-105 \cdot 36^{\circ}$, IR (neat) $1647,1084 \mathrm{~cm}^{-1}$; ${ }^{1} \mathrm{H} \mathrm{NMR}$ :

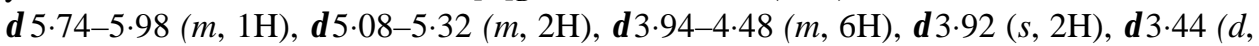
$J=6.8 \mathrm{~Hz}, 1 \mathrm{H}$ ), $\delta 1.53,1.49,1.41,1.36$ (four $s, 4 \times 3 \mathrm{H}$ ); Analysis calc. for $\mathrm{C}_{15} \mathrm{H}_{24} \mathrm{O}_{6}$ : $\mathrm{C}=59.99, \mathrm{H}=8.05$; found: $\mathrm{C}=59.74, \mathrm{H}=7.96$.

2.2h 3-O-allyl-1,2:5,6-di-O-isopropylideneglucofuranose (entry 8): The glucose diacetonide $\left(260 \mathrm{mg}, 1 \mathrm{mmol}\right.$ ) was converted to its allyl ether ${ }^{15}$ in $94 \%$ yield as a colourless oil in $3 \cdot 5 \mathrm{~h} .[\alpha]_{\mathrm{D}}=-25 \cdot 76^{\circ}$, IR (neat) $1640,1160 \mathrm{~cm}^{-1} ;{ }^{1} \mathrm{H}$ NMR: $\delta 5 \cdot 83-5 \cdot 89$ $(m, 1 \mathrm{H}), \delta 5.89(d, J=3.9 \mathrm{~Hz}, 2 \mathrm{H}), \delta 5.23-5.34(m, 2 \mathrm{H}), \delta 4.55(d, J=3.4 \mathrm{~Hz}, 2 \mathrm{H})$, $\delta 4.29-4.34(m, 1 \mathrm{H}), \delta 3.94-4.29(m, 3 \mathrm{H}), \delta 3.83(d, J=9.28 \mathrm{~Hz}, 1 \mathrm{H}), \delta 1.5,1.42,1.35$, $1 \cdot 31$ (four $s, 4 \times 3 \mathrm{H}$ ).

2.2i Allyl phenyl ether (entry 9): Phenol (94 mg, $1 \mathrm{mmol}$ ) was converted to its allyl ether in $89 \%$ yield as a colourless oil in $14 \mathrm{~h}$. IR (neat): $1630 \mathrm{~cm}^{-1},{ }^{1} \mathrm{H}$ NMR: $\delta 7 \cdot 27(\mathrm{~m}$, $5 \mathrm{H}), \delta 5 \cdot 89-6 \cdot 11(m, 1 \mathrm{H}), \delta 5 \cdot 22-5 \cdot 44(m, 2 \mathrm{H}), \delta 4 \cdot 53(m, 2 \mathrm{H})$.

2.2j Allyl p-methylphenyl ether (entry 10): p-Cresol (108 mg, $1 \mathrm{mmol}$ ) was converted to its allyl ether ${ }^{16}$ in $85 \%$ yield as colourless oil. IR (neat): $1600,1100 \mathrm{~cm}^{-1}$; ${ }^{1} \mathrm{H}$ NMR: $\delta 7.08(d, J=7.81 \mathrm{~Hz}, 2 \mathrm{H}), \delta 6.81(d, J=8.79 \mathrm{~Hz}, 2 \mathrm{H}), \delta 6.01-6.08(m, 1 \mathrm{H}), \delta 5 \cdot 28-5.42$ $(m, 2 \mathrm{H}), \delta 4.51(d, J=1.48 \mathrm{~Hz}, 2 \mathrm{H}), \delta 2 \cdot 26(\mathrm{~s}, 3 \mathrm{H})$.

\subsection{Representative procedure for the preparation of benzyl ethers}

2.3a Benzyl decyl ether (entry 1, table 1): Conversion of $n$-decanol to its benzyl ether is given as a representative example. A mixture of $n$-decanol (158 mg, $1 \mathrm{mmol}$ ) and benzyl bromide $(0.36 \mathrm{~mL}, 3 \mathrm{mmol})$ and $\mathrm{KOH}(1 \mathrm{pellet}, \approx 120 \mathrm{mg}, 2 \mathrm{mmol})$ was stirred at room temperature for $18 \mathrm{~h}$ (completion of the reaction was monitored by TLC). The crude mixture was loaded on a pad of silica gel (100-200 mesh, $1 \times 5 \mathrm{~cm}$ column) and eluted with hexanes: ethyl acetate $(98: 2)$ to yield benzyl decyl ether ${ }^{10}$ in $94 \%$ yield. IR (neat): 3067, 3030, 2926, 2855, 1707, 1495, 1454, 1362, 1204, 1103, $1028 \mathrm{~cm}^{-1}$; ${ }^{1} \mathrm{H}$ NMR: $\delta 7.36(s, 5 \mathrm{H}), \delta 4.51(s, 2 \mathrm{H}), \delta 3.46(t, J=12.82 \mathrm{~Hz}, 2 \mathrm{H}), \delta 1.5-1.64(m, 2 \mathrm{H}), \delta 1.3$ (broad $s, 14 \mathrm{H}), \delta 0 \cdot 9(t, J=12 \cdot 4 \mathrm{~Hz}, 3 \mathrm{H})$. 
2.3b Dibenzyl ether (entry 2): Benzyl alcohol (108 mg, $1 \mathrm{mmol}$ ) was converted to dibenzyl ether in $81 \%$ yield in $35 \mathrm{~h}$. IR (neat): $3033,2858,1549 \mathrm{~cm}^{-1} ;{ }^{1} \mathrm{H}$ NMR: $\delta 7.4(s$, $10 \mathrm{H}), \delta 4.56(s, 4 \mathrm{H})$.

2.3c Benzyl geranyl ether (entry 3): Geraniol (154 mg, $1 \mathrm{mmol}$ ) was converted to its benzyl ether ${ }^{17}$ in $91 \%$ yield in $18 \mathrm{~h}$. IR (neat): 1640,$1595 ;{ }^{1} \mathrm{H}$ NMR: $\delta 7.20(s, 5 \mathrm{H})$, $\delta 5.33($ br t $, J=7 \mathrm{~Hz}, 1 \mathrm{H}), \delta 5.03(s, 1 \mathrm{H}), \delta 4.40(s, 2 \mathrm{H}), \delta 3.92(d, J=7 \mathrm{~Hz}, 2 \mathrm{H}), \delta 2.01$ $(m, 4 \mathrm{H}), \delta 1.65(s, 3 \mathrm{H}), 1.62(s, 6 \mathrm{H})$.

2.3d Benzyl neryl ether (entry 4): Nerol (154 mg, $1 \mathrm{mmol}$ ) was converted to its benzyl ether ${ }^{13}$ in $91 \%$ yield in $20 \mathrm{~h}$. IR (neat) $1640,1600 \mathrm{~cm}^{-1} ;{ }^{1} \mathrm{H}$ NMR: $\delta 7 \cdot 25(s, 5 \mathrm{H}), 5 \cdot 21$ $(m, 2 \mathrm{H}), \delta 4.42(s, 2 \mathrm{H}), \delta 3.94(d, J=6 \mathrm{~Hz}, 2 \mathrm{H}), \delta 2.03(b r d, J=3 \mathrm{~Hz}, 4 \mathrm{H}), \delta 1.76(b r s$, $3 \mathrm{H}) 1.67,1.58$ (two $s, 2 \times 3 \mathrm{H})$.

2.3e Benzyl cholesteryl ether (entry 6): Cholesterol (100 mg, 0.26 mmol) was converted to its benzyl ether ${ }^{18}$ in $86 \%$ yield in $16 \mathrm{~h} .[\alpha]_{\mathrm{D}}=-5.11^{\circ},{ }^{1} \mathrm{H}$ NMR: $\delta 7 \cdot 3(\mathrm{~m}, 5 \mathrm{H}), \delta 5 \cdot 35$ $(d, J=6.66 \mathrm{~Hz}, 1 \mathrm{H}), \delta 4.54(s, 2 \mathrm{H}), \delta 2.2-3.3(m, 1 \mathrm{H}), \delta 0.77-2.07(m, 28 \mathrm{H}), \delta 1.0(s$, $3 \mathrm{H}), \delta 0 \cdot 77-0.93(b r d, 9 \mathrm{H}), \delta 0 \cdot 66(s, 3 \mathrm{H})$.

$2.3 f$ 3-O-Benzyl-1,2:4,5-di-O-isopropylidenefructopyranose (entry 7): Fructose diacetonide (200 mg, $0.77 \mathrm{mmol}$ ) was converted to its benzyl ether ${ }^{19}$ in $94 \%$ yield as colourless syrup in $20 \mathrm{~h} .[\alpha]_{\mathrm{D}}=-92.91^{\circ}$, IR (neat): 3026, 2939, 2892, 2361, 1595, 1461, 1428, 1381, 1327, 1219, 1119, 1085, 1024, 978, 897, $776 \mathrm{~cm}^{-1} ;{ }^{1} \mathrm{H}$ NMR: $\delta 7 \cdot 34(m, 5 \mathrm{H})$, $\delta 5.0(d, J=11.97 \mathrm{~Hz}, 1 \mathrm{H}), \delta 4.7(d, J=11.98 \mathrm{~Hz}, 1 \mathrm{H}), \delta 4.4(t, J=12.78 \mathrm{~Hz}, 1 \mathrm{H})$, $\delta 3.86-4.25(m, 3 \mathrm{H}), \delta 4.07(s, 2 \mathrm{H}), \delta 3.49(d, J=7.23 \mathrm{~Hz}, 1 \mathrm{H}), \delta 1.55,1.5,1.43,1.39$ (four $s, 4 \times 3 \mathrm{H}$ ).

$2.3 \mathrm{~g}$ 3-O-Benzyl-1,2:5,6-di-O-isopropylideneglucofuranose (entry 8): The glucose diacetonide $\left(250 \mathrm{mg}, 0.96 \mathrm{mmol}\right.$ ) was converted to its benzyl ether ${ }^{20}$ in $92 \%$ yield as colourless syrup in $36 \mathrm{~h} .[\alpha]_{\mathrm{D}}=-23.33^{\circ}$, IR (neat): 3026, 2361, 1596, 1428, 1374, 1219, 1078, 1025, 863, 763, $675 \mathrm{~cm}^{-1}$; ${ }^{1} \mathrm{H}$ NMR: $\delta 7.4(m, 5 \mathrm{H}), \delta 5.9(d, J=3.61 \mathrm{~Hz}, 1 \mathrm{H})$, $\delta 4.65(d, J=4.88 \mathrm{~Hz}, 1 \mathrm{H}), \delta 4.55(s, 2 \mathrm{H}), \delta 4.27-4.42(m, 1 \mathrm{H}), \delta 3.97-4.21(m, 3 \mathrm{H})$, $\delta 3.7(m, 1 \mathrm{H}), \delta 1.49,1.43,1.37,1.31$ (four $s, 4 \times 3 \mathrm{H})$.

\section{Acknowledgement}

HSPR thanks the University Grants Commission, New Delhi for financial support.

\section{References}

1. Corey E J and Suggs W J 1973 J. Org. Chem. 383224

2. Oltvoort J T, Kloosterman M and Van Boom J H 1983 J. R. Neth. Chem. Soc.102 501

3. Guibe F and M'Leux Y S 1981 Tetrahedron Lett. 223591

4. Lakhmiri R, Lhoste P and Sinou D 1989 Tetrahedron Lett. 304669

5. Kahn R, Low I and Trishman H 1957 Chem. Ber. 90203

6. van Tamelen E E, Zawacky S R, Russell R K and Carlson J G 1983 J. Am. Chem. Soc. 105142

7. Liotta L J and Ganem B 1989 Tetrahedron Lett. 304759

8. Surya Prakash Rao H, Senthilkumar S P, Srinivasa Reddy D and Mehta G 1999 Indian J. Chem. B38 260 
9. Whistler R L, Wolfrom M L and Be'Miller J M 1963 Methods in carbohydrate chemistry (New York and London: Academic Press) vol. 2, p. 166

10. Bogdal D, Pielichowski J and Jaskot K 1998 Org. Prep. Proc. Int. 30427

11. Cookson R C and Wallis S R 1966 J. Chem. Soc. 1245

12. Cerichelli C, Freddi A, Loero M A, Pellacani L and Tardella P A 1992 Tetrahedron 482495

13. Alonso E and Ramon D J 1997 Tetrahedron 5314355

14. Ziegler F E, Brown E G and Sobolov S B 1990 J. Org. Chem. 553691

15. Khan A R, Tripathi, R P and Bhaduri A P 1996 Indian J. Chem. B35 405

16. Satyanarayana V, Rao Ch P and Krupadanam G L D 1991 Syn. Commun. 141455

17. Araki S and Butsugan Y 1984 J. Chem. Soc., Perkin Trans. 1969

18. Sarma J C, Borbaruah M, Sarma D N, Barua, N C and Sarma R P 1986 Tetrahedron 423999

19. Kang J, Lim J G, Yoon, S K and Kim M Y 1995 J. Org. Chem. 60, 564

20. Heathcock C H, White C T, Morrison J J and Van Denveer D 1981 J. Org. Chem. 461296 